\title{
Erratum to: Existence and stability of symmetric periodic simultaneous binary collision orbits in the planar pairwise symmetric four-body problem
}

\author{
Lennard F. Bakker • Tiancheng Ouyang • \\ Duokui Yan · Skyler Simmons
}

Published online: 31 March 2012

(C) Springer Science+Business Media B.V. 2012

\section{Erratum to: Celest Mech Dyn Astr (2011) 110:271-290 DOI 10.1007/s10569-011-9358-y}

The conclusions of the numerical results on the linear stability of the periodic orbits remain valid for $0.27<m \leq 1$ where the error in the numerical estimate of the repeated trivial characteristic multiplier 1 remains small. However, there is an error in the stated algebraic multiplicity of the trivial characteristic multiplier 1 for each periodic orbit. Instead of at least two, it should be at least four.

In Lemma 3.1 the final sentence should read as: "The angular momentum A and the $\mathrm{SO}(2)$ symmetry of the PPS4BP contribute to the algebraic multiplicity of the trivial characteristic multiplier 1 of $\gamma_{\epsilon}(s)$. Proving the following result is routine (see Meyer and Hall 1992)."

Lemma 3.2 should read as: If $\gamma(s)$ is a periodic orbit of the Hamiltonian system of equations with Hamiltonian $\hat{\Gamma}$ on the level set $\hat{\Gamma}=0$, then for each $\epsilon>0$ the periodic orbit $\gamma_{\epsilon}(s)$ has 1 as a defective characteristic multiplier with algebraic multiplicity at least four.

For each $\epsilon>0$, the periodic orbit $\gamma_{\epsilon}(s)$ is spectrally stable if all of its characteristic multipliers have modulus one. By Lemma 3.2, the periodic orbit $\gamma_{\epsilon}(s)$ has 1 as

The online version of the original article can be found under doi:10.1007/s10569-011-9358-y.

L. F. Bakker $(\varangle) \cdot$ T. Ouyang $\cdot$ S. Simmons

Department of Mathematics, Brigham Young University, Provo, UT 84602, USA

e-mail: bakker@math.byu.edu

T. Ouyang

e-mail: ouyang@math.byu.edu

S. Simmons

e-mail: xinkaisen@gmail.com

D. Yan

Chern Institute of Mathematics, Nankai University, Tianjin 300071, People's Republic of China

e-mail: duokuiyan@gmail.com 
a defective characteristic multiplier with algebraic multiplicity at least four, and so the monodromy matrix $X_{\epsilon}\left(T_{\epsilon}\right)$ is not semisimple. However, there is a four-dimensional subspace $U_{1}$ containing

$$
\operatorname{Span}\left(\gamma_{\epsilon}^{\prime}(0),\left(\frac{\partial}{\partial \epsilon} \gamma_{\epsilon}\right)(0)\right)
$$

that is $X_{\epsilon}\left(T_{\epsilon}\right)$-invariant. The periodic orbit $\gamma_{\epsilon}(s)$ is said to be linearly stable if it is spectrally stable and there exists a 4-dimensional $X_{\epsilon}\left(T_{\epsilon}\right)$-invariant subspace $U_{2}$ such that $U_{1}+U_{2}=\mathbb{R}^{8}$ and $X_{\epsilon}\left(T_{\epsilon}\right)$ restricted to $U_{2}$ is semisimple. A proof of the following is routine.

Theorem 3.3 Suppose $\gamma(s)$ is a periodic orbit of the Hamiltonian system of equations with Hamiltonian $\hat{\Gamma}$ on the level set $\hat{\Gamma}=0$. Then $\gamma_{\epsilon}(s)$ is spectrally (linearly) stable for some $\epsilon>0$ if and only if $\gamma_{\epsilon}(s)$ is spectrally (linearly) stable for all $\epsilon>0$.

In the heading 6 Numerical estimates in the equal mass case, the paragraph should read as: The components of the scaled periodic orbit $\gamma_{\epsilon}(s)$ for an $\epsilon>1$, shown in Fig. 2, satisfy the symmetries $S_{F} \gamma_{\epsilon}(s)=\gamma_{\epsilon}\left(s+T_{\epsilon} / 4\right)$ and $S_{G} \gamma_{\epsilon}(s)=\gamma_{\epsilon}\left(T_{\epsilon} / 4-s\right)$. We choose $\epsilon$ so that $T_{\epsilon}=2 \pi$, and check the linear stability of $\gamma_{\epsilon}(s)$ (see Theorem 3.3). Using a RungeKutta order 4 algorithm with a fixed time step of $2 \pi / 50000$, we computed $X_{\epsilon}(2 \pi)$. Four of the eigenvalues of $X_{\epsilon}(2 \pi)$ are 1 by Theorem 3.2. Numerical estimates of the remaining eigenvalues of $X_{\epsilon}(2 \pi)$ are

$$
\begin{aligned}
& -0.9888731375 \pm 0.1487612779 i, \\
& -0.9973584383 \pm 0.07263708002 i,
\end{aligned}
$$

that have modulus one. Thus numerically, the periodic orbits $\gamma_{\epsilon}(s)$ are linearly stable for all $\epsilon>0$. The first complex conjugate pair of eigenvalues for $X_{\epsilon}(2 \pi)$ matches the complex conjugate pair of characteristic multipliers for the periodic orbit $Q_{1}(\sigma), Q_{2}(\sigma), P_{1}(\sigma), P_{2}(\sigma)$ of the Hamiltonian system of equations (14) with Hamiltonian $\Gamma$, corresponding to $\gamma_{1}(s)=$ $\gamma(s)$, where we computed (Bakker et al. 2010) the real part of the complex conjugate pair of modulus one to be -0.9888840619 . Because, by a lengthy computation, $J \nabla^{2} \hat{\Gamma}\left(\gamma_{\epsilon}(s)\right)$ is not block diagonal, the second complex conjugate pair of eigenvalues of $X_{\epsilon}(2 \pi)$ are not repeats of the characteristic multipliers of the periodic orbit $Q_{1}(\sigma), Q_{2}(\sigma), P_{1}(\sigma), P_{2}(\sigma)$.

In the heading 7 Numerical estimates in the unequal mass case, the final paragraph should read as: We numerically estimate the monodromy matrices $X(2 \pi ; m)$ and their eigenvalues for $\gamma(s ; m)$ with $0<m \leq 1$. We used a Runge-Kutta order 4 algorithm coded in MATLAB with a fixed time step of $2 \pi / 50000$ at increments of 0.01 in $m$. In Fig. 6 we graph the maximum modulus of the eight eigenvalues of $X(2 \pi ; m)$ over $0.2<m \leq 1$. The maximum modulus of the eigenvalues of $X(2 \pi ; m)$ for $0<m \leq 0.2$ becomes large as $m \rightarrow 0$, and are therefore not included in Fig. 6 . The maximum modulus for the eight eigenvalues shows that $\gamma(s ; m)$ is linearly unstable for $0<m \leq 0.53$. The error in the numerical estimation of the repeated eigenvalue 1 of $X(2 \pi ; m)$ is large enough to account for the graph in Fig. 6 not lying at 1 over $0.54 \leq m \leq 1$. However, for $0.54 \leq m \leq 1$, the other four eigenvalues come in two distinct complex conjugate pairs that are each of modulus one and located away from \pm 1 . This shows that $\gamma(s ; m)$ is linearly stable for $0.54 \leq m \leq 1$. 